\title{
Supramolecular Hydrogels Hybridized with Single-Walled Carbon Nanotubes
}

\author{
Zhimin Wang, Yongming Chen ${ }^{*}$
}

State Key Laboratory of Polymer Physics and Chemistry, Institute of Chemistry, The Chinese Academy of Sciences, Beijing 100080, China.

Supporting Information.

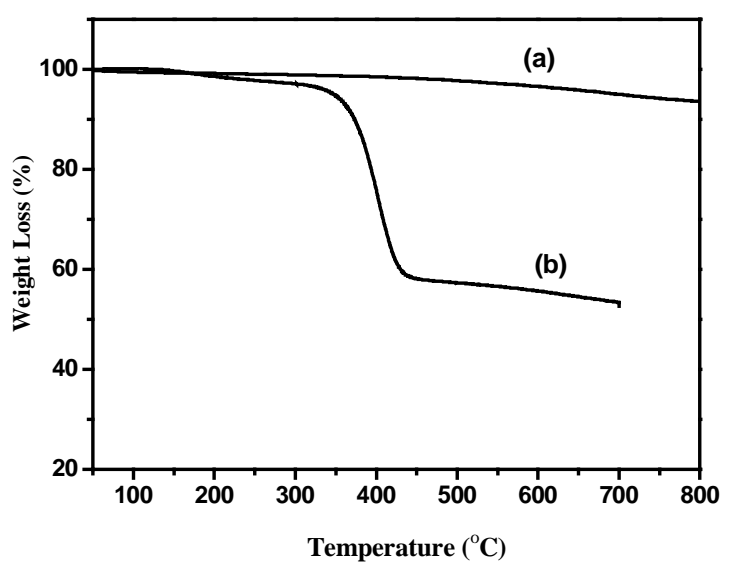

Figure S1. Thermogravimetric analysis of (a) pristine SWNTs and (b) centrifuged precipitate for determining the exact concentrations of SWNTs and Pluronic copolymer in solution.

\footnotetext{
* To whom correspondence should be addressed: E-mail: ymchen@iccas.ac.cn
} 


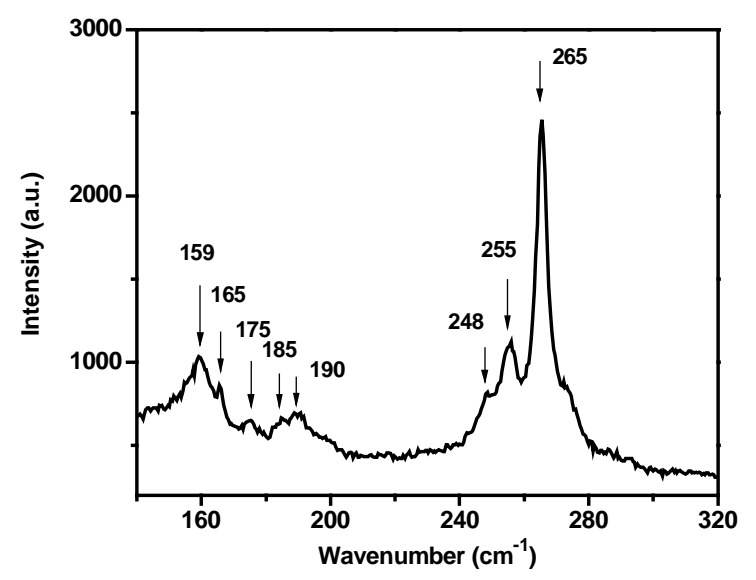

Figure S2. Magnified view of the radial breathing model of SWNTs dispersed in the hybrid hydrogel.

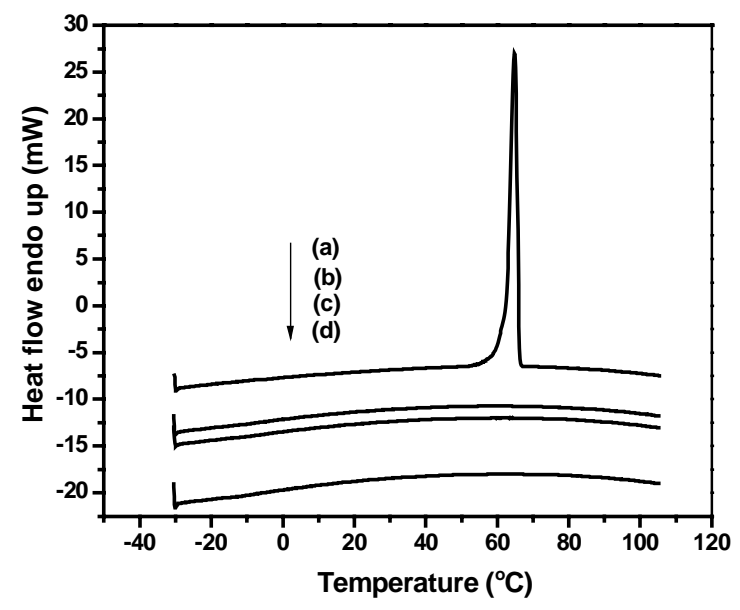

Figure S3. DSC traces of (a) Pluronic copolymer, (b) native hydrogel, (c) pure $\alpha$-CD and (d) hybrid hydrogel. 


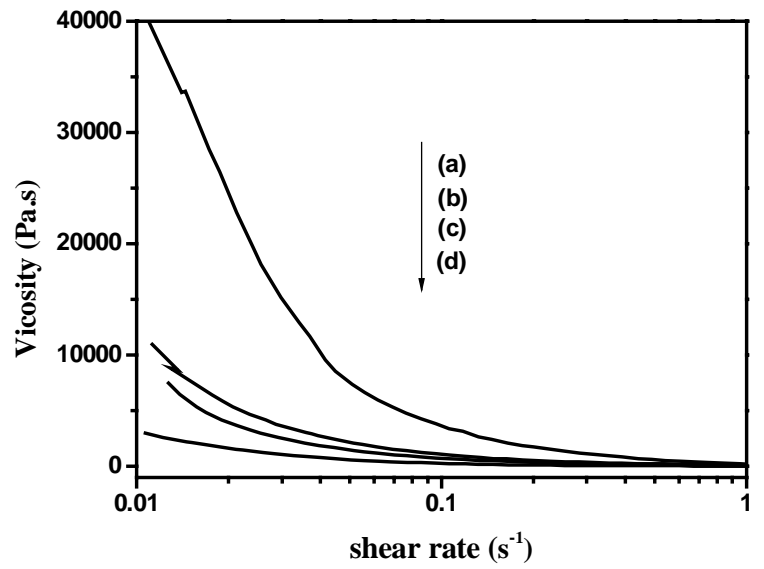

Figure S4 $\square$ The influence of SWNTs on the steady flow behavior of supramolecular hydrogels. (a) native hydrogel; (b) $0.01 \mathrm{wt} \%$ SWNTs in hydrogel; (c) $0.05 \mathrm{wt} \%$ SWNTs in hydrogel; (d) $0.2 \mathrm{wt} \%$ SWNTs in hydrogel.

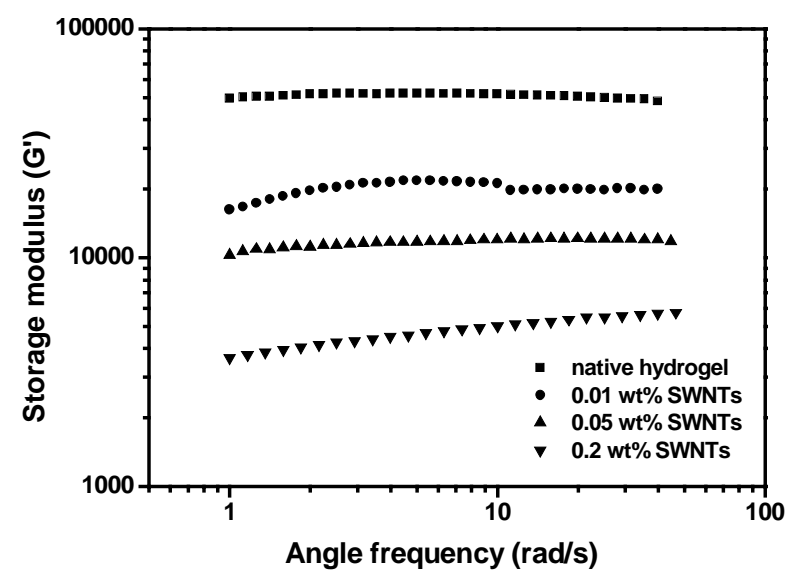

Figure S5. Influence of SWNTs contents on storage modulus of supramolecular hydrogels. 


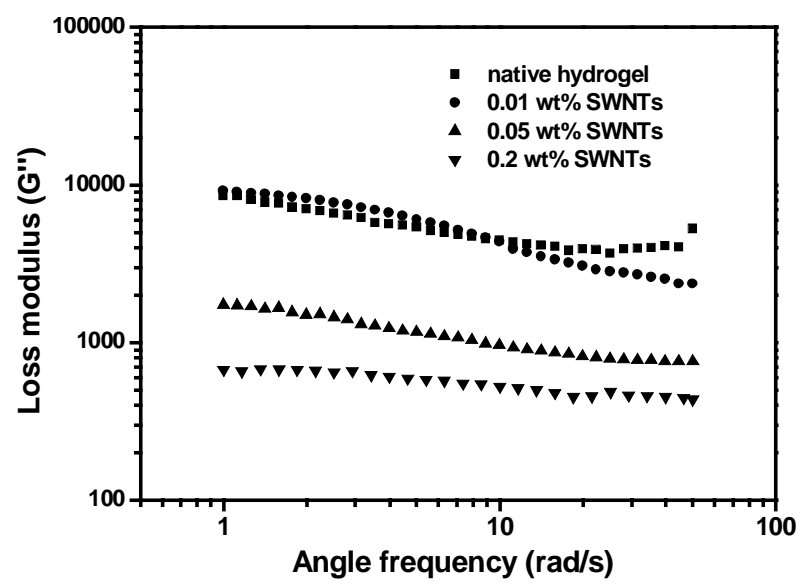

Figure S6. Influence of SWNTs contents on loss modulus of supramolecular hydrogels. 